\title{
MULTIPLICATION OPERATORS ON THE BLOCH SPACE OF BOUNDED HOMOGENEOUS DOMAINS
}

\author{
ROBERT F. ALLEN AND FLAVIA COLONNA
}

\begin{abstract}
In this paper, we study the multiplication operators on the Bloch space of a bounded homogeneous domain in $\mathbb{C}^{n}$. Specifically, we characterize the bounded and the compact multiplication operators, establish estimates on the operator norm, and determine the spectrum. Furthermore, we prove that for a large class of bounded symmetric domains, the isometric multiplication operators are those whose symbol is a constant of modulus one.
\end{abstract}

\section{INTRODUCTION}

In recent years, the operator theory of many functional Banach spaces that arise in complex function theory has been studied extensively. Two particular important classes of operators are the multiplication operators and the composition operators. While a great deal is known about the composition operators on a wide variety of functional Banach spaces (see [17] and [9]), in the literature the amount on the study of multiplication operators on some spaces has been surprisingly small.

Multiplication operators on the Bloch space of the open unit disk $\mathbb{D}$ have been studied in [3], 4], 15], and [1. In this setting, criteria for the boundedness of multiplications operators were obtained independently by Arazy [3], and by Brown and Shields 4. More recently, Ohno and Zhao characterized the bounded and the compact weighted composition operators on the Bloch space and on its subspace given by the closure of the polynomials known as the little Bloch space [15. As a corollary, they deduced that the only compact multiplication operators are those whose symbol is identically zero. In [1, the authors gave estimates on the operator norm, determined the spectrum, and showed that the only isometric multiplication operators are those whose symbol is a unimodular constant.

Bloch functions in higher dimensions have been introduced on bounded homogeneous domains in 12. Krantz and Ma defined the notion of Bloch function on a strongly pseudoconvex domain [14].

The study of the multiplication operators on the Bloch space in higher dimensions was begun by Zhu, who characterized the bounded multiplication operators on the Bloch space and the little Bloch space of the unit ball $\mathbb{B}_{n}[23$. In [21] and 22. Zhou and Chen characterized the bounded and the compact weighted composition operators on the Bloch space of the unit ball and the unit polydisk, thereby obtaining corresponding results for the multiplication operators.

Date: November 24, 2018.

1991 Mathematics Subject Classification. Primary 47B35; Secondary 32A18.

Key words and phrases. Multiplication operators, Bloch space, Homogeneous domains. 
In this work, we carry out the study of the multiplication operators on the Bloch space of a bounded homogeneous domain and on a subspace we call the *little Bloch space. Specifically, we extend the results of Zhu, Zhao and Chen, and obtain operator norm estimates similar to those found for the case of the unit disk. Furthermore, we determine the spectrum and show that for the bounded symmetric domains that do not have the disk as a factor, the only isometric multiplication operators are those whose symbol is a constant function of modulus one.

\section{Preliminaries}

A domain $D \subset \mathbb{C}^{n}$ is homogeneous if the group $\operatorname{Aut}(D)$ of biholomorphic selfmaps of $D$ (which we call automorphisms) acts transitively on $D$. An important subclass of homogeneous domains are the symmetric domains. A domain $D \subset \mathbb{C}^{n}$ is symmetric at $z_{0} \in D$ if there exists an involutive automorphism of $D$ for which $z_{0}$ is an isolated fixed point. A domain $D$ is said to be symmetric if $D$ is symmetric at each point.

Cartan [5] showed that every bounded symmetric domain in $\mathbb{C}^{n}$ is biholomorphic to a finite product of irreducible bounded symmetric domains, unique up to order. Moreover, Cartan classified the irreducible bounded symmetric domains into six classes; four of the classes are referred to as Cartan classical domains, whereas the other two, each consisting of a single domain, are referred to as the exceptional domains. A bounded symmetric domain written as such a product is said to be in standard form. The Cartan classical domains are defined as:

$$
\begin{aligned}
R_{I} & =\left\{Z \in M_{m, n}(\mathbb{C}): I_{m}-Z Z^{*}>0\right\}, \text { for } m \geq n \geq 1, \\
R_{I I} & =\left\{Z \in M_{n}(\mathbb{C}): Z=Z^{T}, I_{n}-Z Z^{*}>0\right\}, \text { for } n \geq 1, \\
R_{I I I} & =\left\{Z \in M_{n}(\mathbb{C}): Z=-Z^{T}, I_{n}-Z Z^{*}>0\right\}, \text { for } n \geq 2, \\
R_{I V} & =\left\{z=\left(z_{1}, \ldots, z_{n}\right) \in \mathbb{C}^{n}: A>0,\|z\|^{2}<1\right\}, \text { for } 1 \leq n \neq 2,
\end{aligned}
$$

where $M_{m, n}(\mathbb{C})$ denotes the set of $m \times n$ matrices with entries in $\mathbb{C}, M_{n}(\mathbb{C})=$ $M_{n, n}(\mathbb{C}), Z^{T}$ is the transpose of $Z, A=\left|\sum z_{j}^{2}\right|^{2}+1-2\|z\|^{2}$, and $\|z\|^{2}=\sum\left|z_{j}\right|^{2}$. To assure these classes are disjoint, the dimensional restrictions $n \geq 2$ for domains in $R_{I I}$ and $n \geq 5$ for domains in $R_{I I I}$ and $R_{I V}$ are imposed. For a description of the exceptional domains we refer the reader to [10].

Remark 1. Every bounded symmetric domain is homogeneous and a bounded homogeneous domain that is symmetric at a single point is symmetric. Each bounded homogeneous domain $D$ is endowed with a canonical metric invariant under the action of $\operatorname{Aut}(D)$, called the Bergman metric. The unit ball $\mathbb{B}_{n}$ and the unit polydisk $\mathbb{D}^{n}$ are examples of bounded symmetric domains in $\mathbb{C}^{n}$. A description of the Bergman metric and the automorphism group of the Cartan classical domains can be found in 13 . Cartan proved that every bounded homogeneous domain in dimensions 2 and 3 is symmetric. An example of a homogeneous domain in dimension 4 that is not symmetric can be found in [16].

In 12] Hahn introduced the notion of Bloch function on a bounded homogeneous domain. In [18] and [19] Timoney expanded the study of Bloch functions in this setting. In this article, we adopt the terminology and notation used by Timoney. We denote by $H(D)$ the set of holomorphic functions from $D \subset \mathbb{C}^{n}$ into $\mathbb{C}$ and by $H^{\infty}(D)$ the set of bounded functions in $H(D)$. 
Let $D$ be a bounded homogeneous domain in $\mathbb{C}^{n}$ and $f \in H(D)$. Then for $z \in D$, define

$$
Q_{f}(z)=\sup _{u \in \mathbb{C}^{n} \backslash\{0\}} \frac{|\nabla(f)(z) u|}{H_{z}(u, \bar{u})^{1 / 2}}
$$

where for $u=\left(u_{1}, \ldots, u_{n}\right) \in \mathbb{C}^{n}, \nabla(f)(z) u=\sum_{j=1}^{n} \frac{\partial f}{\partial z_{j}}(z) u_{j}$, and $H_{z}(\cdot, \cdot)$ is the Bergman metric of $D$. A function $f$ is called Bloch if

$$
\beta_{f}=\sup _{z \in D} Q_{f}(z)<\infty .
$$

It was shown by Timoney that every bounded holomorphic function on $D$ is Bloch (see [18, Example 3.7(2)). For a fixed $z_{0} \in D$, the Bloch space on $D$ based at $z_{0}$ is the Banach space $\mathcal{B}(D)$ of all Bloch functions on $D$ under the norm

$$
\|f\|_{\mathcal{B}}=\left|f\left(z_{0}\right)\right|+\beta_{f} \text {. }
$$

For convenience, we shall assume that $D$ contains the origin and choose 0 as the base point to define the norm. Note that if $D=\mathbb{D}, Q_{f}(z)=\left(1-|z|^{2}\right)\left|f^{\prime}(z)\right|$.

In [19] the little Bloch space on the unit ball was defined as

$$
\mathcal{B}_{0}\left(\mathbb{B}_{n}\right)=\left\{f \in \mathcal{B}\left(\mathbb{B}_{n}\right): \lim _{\|z\| \rightarrow 1^{-}} Q_{f}(z)=0\right\}
$$

which is the closure of the polynomials in $\mathcal{B}\left(\mathbb{B}_{n}\right)$. However, when $D$ is a bounded symmetric domain other than the ball, the only Bloch functions $f$ on $D$ such that $Q_{f}(z) \rightarrow 0$ as $z$ approaches the boundary $\partial D$ of $D$ are the constants, so the little Bloch space $\mathcal{B}_{0}(D)$ is defined as the closure of the polynomials.

The *-little Bloch space is defined as

$$
\mathcal{B}_{0^{*}}(D)=\left\{f \in H(D): \lim _{z \rightarrow \partial^{*} D} Q_{f}(z)=0\right\},
$$

where $\partial^{*} D$ denotes the distinguished boundary of $D$. If $D$ is the unit ball, then $\partial D=\partial^{*} D$ and thus $\mathcal{B}_{0}(D)=\mathcal{B}_{0^{*}}(D)$, while when $D \neq \mathbb{B}_{n}, \mathcal{B}_{0}(D)$ is a proper subspace of $\mathcal{B}_{0^{*}}(D)$, and $\mathcal{B}_{0^{*}}(D)$ is a non-separable subspace of $\mathcal{B}(D)$.

\section{Boundedness}

Let $D$ be a bounded homogeneous domain in $\mathbb{C}^{n}$. For $z \in D$, we define

$$
\begin{gathered}
\omega(z)=\sup _{f \in \mathcal{B}(D)}\left\{|f(z)|: f(0)=0 \text { and }\|f\|_{\mathcal{B}} \leq 1\right\}, \\
\omega_{0}(z)=\sup _{f \in \mathcal{B}_{0^{*}}(D)}\left\{|f(z)|: f(0)=0 \text { and }\|f\|_{\mathcal{B}} \leq 1\right\} .
\end{gathered}
$$

In 2] it was shown that if $f \in \mathcal{B}(D)$, then

$$
\beta_{f}=\sup _{z \neq w} \frac{|f(z)-f(w)|}{\rho(z, w)}
$$

where $\rho$ is the distance induced by the Bergman metric on $D$. Consequently, if $f \in \mathcal{B}(D)$ with $f(0)=0$ and $\|f\|_{\mathcal{B}} \leq 1$, then $|f(z)| \leq \rho(z, 0)$ for each $z \in D$. Thus

$$
\omega_{0}(z) \leq \omega(z) \leq \rho(z, 0),
$$

and hence $\omega_{0}(z)$ and $\omega(z)$ are finite for all $z \in D$.

Lemma 3.1. Let $D$ be a bounded homogeneous domain in $\mathbb{C}^{n}$ and $z \in D$. 
(a) If $f \in \mathcal{B}(D)$, then

$$
|f(z)| \leq|f(0)|+\omega(z) \beta_{f}
$$

(b) If $f \in \mathcal{B}_{0^{*}}(D)$, then

$$
|f(z)| \leq|f(0)|+\omega_{0}(z) \beta_{f} .
$$

Proof. Suppose $f \in \mathcal{B}(D)$ is not constant. Then the function $g$ defined by $g(z)=$ $\frac{1}{\beta_{f}}(f(z)-f(0))$ is holomorphic and such that $g(0)=0$ and $\|g\|_{\mathcal{B}}=1$. So $|g(z)| \leq$ $\omega(z)$ for all $z \in D$. Thus,

$$
|f(z)| \leq|f(0)|+|f(z)-f(0)|=|f(0)|+|g(z)| \beta_{f} \leq|f(0)|+\omega(z) \beta_{f},
$$

for all $z \in D$. The result for $\mathcal{B}_{0^{*}}(D)$ is analogous.

For $\psi \in H(D)$, define

$$
\begin{aligned}
\sigma_{\psi} & =\sup _{z \in D} \omega(z) Q_{\psi}(z), \\
\sigma_{0, \psi} & =\sup _{z \in D} \omega_{0}(z) Q_{\psi}(z) .
\end{aligned}
$$

Theorem 3.2. Let $D$ be a bounded homogeneous domain in $\mathbb{C}^{n}$ and $\psi \in H(D)$.

(a) $M_{\psi}$ is bounded on $\mathcal{B}(D)$ if and only if $\psi \in H^{\infty}(D)$ and $\sigma_{\psi}<\infty$.

(b) $M_{\psi}$ is bounded on $\mathcal{B}_{0^{*}}(D)$ if and only if $\psi \in H^{\infty}(D) \cap \mathcal{B}_{0^{*}}(D)$ and $\sigma_{0, \psi}<$ $\infty$.

Proof. To prove (a), assume $\psi \in H^{\infty}(D)$ with $\sigma_{\psi}<\infty$, and let $f \in \mathcal{B}(D)$. Applying the product rule, for all $z \in D$ we have

$$
Q_{\psi f}(z) \leq|\psi(z)| Q_{f}(z)+|f(z)| Q_{\psi}(z) .
$$

Since $\psi$ is bounded, using Lemma 3.1(a), inequality (3) yields

$$
\begin{aligned}
\beta_{\psi f} & \leq\|\psi\|_{\infty} \beta_{f}+\sup _{z \in D}\left(|f(0)|+\omega(z) \beta_{f}\right) Q_{\psi}(z) \\
& \leq\|\psi\|_{\infty} \beta_{f}+|f(0)| \beta_{\psi}+\sigma_{\psi} \beta_{f},
\end{aligned}
$$

which is finite. Thus, $M_{\psi} f \in \mathcal{B}(D)$ and

$$
\left\|M_{\psi} f\right\|_{\mathcal{B}} \leq|f(0)|\|\psi\|_{\mathcal{B}}+\left(\|\psi\|_{\infty}+\sigma_{\psi}\right) \beta_{f} .
$$

It follows immediately that $\left\|M_{\psi} f\right\|_{\mathcal{B}} \leq\left(\|\psi\|_{\mathcal{B}}+\|\psi\|_{\infty}+\sigma_{\psi}\right)\|f\|_{\mathcal{B}}$, proving that $M_{\psi}$ is bounded on $\mathcal{B}(D)$.

Conversely, suppose $M_{\psi}$ is bounded on $\mathcal{B}(D)$. By Lemma 11 of [11, $\psi \in H^{\infty}(D)$ with $\|\psi\|_{\infty} \leq\left\|M_{\psi}\right\|$. Thus, it suffices to show that $\sigma_{\psi}$ is finite. Let $f \in \mathcal{B}(D)$. Applying the product rule, for each $z \in D$ we obtain

$$
\begin{aligned}
|f(z)| Q_{\psi}(z) & \leq Q_{\psi f}(z)+|\psi(z)| Q_{f}(z) \leq\left\|M_{\psi} f\right\|_{\mathcal{B}}+|\psi(z)| Q_{f}(z) \\
& \leq\left(\left\|M_{\psi}\right\|+|\psi(z)|\right)\|f\|_{\mathcal{B}} .
\end{aligned}
$$

Taking the supremum over all Bloch functions $f$ such that $f(0)=0$ and $\|f\|_{\mathcal{B}} \leq 1$, we get $\omega(z) Q_{\psi}(z) \leq\left\|M_{\psi}\right\|+|\psi(z)|$. Finally, taking the supremum over all $z \in D$, we arrive at $\sigma_{\psi} \leq\left\|M_{\psi}\right\|+\|\psi\|_{\infty}$, which is finite.

Next, assume $\psi \in H^{\infty}(D) \cap \mathcal{B}_{0^{*}}(D)$ and $\sigma_{0, \phi}<\infty$. Then for $f \in \mathcal{B}_{0^{*}}(D)$ and $z \in D$, we have $Q_{\psi f}(z) \leq\|\psi\|_{\infty} Q_{f}(z)+\left(|f(0)|+\omega_{0}(z) \beta_{f}\right) Q_{\psi}(z) \rightarrow 0$ as $z \rightarrow \partial^{*} D$. The boundedness of $M_{\psi}$ on $\mathcal{B}_{0^{*}}(D)$ follows by using the argument in the proof of part (a) and Lemma 3.1(b). 
Conversely, if $M_{\psi}$ is bounded on $\mathcal{B}_{0^{*}}(D)$, then $\psi=M_{\psi}(1) \in \mathcal{B}_{0^{*}}(D)$ since $1 \in \mathcal{B}_{0^{*}}(D)$. Arguing as in the proof of part (a), we see that $\sigma_{0, \psi} \leq\left\|M_{\psi}\right\|+\|\psi\|_{\infty}$, completing the proof.

3.1. Equivalence of Boundedness on $\mathcal{B}(D)$ and $\mathcal{B}_{0^{*}}(D)$. We will now discuss conditions for which the boundedness of $M_{\psi}$ is equivalent on the Bloch space and *little Bloch space. We first prove that for the cases of the unit ball and polydisk, the boundedness of $M_{\psi}$ on $\mathcal{B}(D)$ is equivalent to the boundedness on $\mathcal{B}_{0^{*}}(D)$. We then show sufficient conditions for which this is true on a general bounded homogeneous domain.

In the case of the unit ball, the quantities $\omega(z)$ and $\omega_{0}(z)$ are equal to the Bergman distance from 0 to $z$ for all $z \in \mathbb{B}_{n}$, and we have the explicit formula

$$
\omega_{0}(z)=\omega(z)=\frac{1}{2} \log \frac{1+\|z\|}{1-\|z\|}
$$

(see [23], Theorems 3.9 and 3.14). In turn, this implies that $\sigma_{0, \psi}=\sigma_{\psi}$. So, we have the following characterization of bounded multiplication operators on the Bloch space and little Bloch space of the unit ball. Recall that for the unit ball the little Bloch space and the $*$-little Bloch space are the same.

Corollary 3.3. Let $\psi \in H\left(\mathbb{B}_{n}\right)$. Then the following are equivalent:

(a) $M_{\psi}$ is bounded on $\mathcal{B}\left(\mathbb{B}_{n}\right)$.

(b) $M_{\psi}$ is bounded on $\mathcal{B}_{0}\left(\mathbb{B}_{n}\right)$.

(c) $\psi \in H^{\infty}\left(\mathbb{B}_{n}\right)$ and $\sup _{z \in \mathbb{B}_{n}} \log \frac{1+\|z\|}{1-\|z\|} Q_{\psi}(z)$ is finite.

Proof. The equivalence $(a) \Longleftrightarrow(c)$ and the implication $(b) \Longrightarrow(c)$ follow immediately from Theorem 3.2 and (5). To show that $(c) \Longrightarrow(b)$, it suffices to show that $\psi \in \mathcal{B}_{0}\left(\mathbb{B}_{n}\right)$. Since $\sup _{z \in \mathbb{B}_{n}} \log \frac{1+\|z\|}{1-\|z\|} Q_{\psi}(z)$ is finite, and as $\|z\| \rightarrow 1^{-}, \log \frac{1+\|z\|}{1-|| z \mid}$ goes to $\infty$, it must be the case that $Q_{\psi}(z) \rightarrow 0$. Thus $\psi \in \mathcal{B}_{0}\left(\mathbb{B}_{n}\right)$, as desired.

We will now show the analogous result for the Bloch space on the polydisk. We first need the following lemma.

Lemma 3.4. For $z \in \mathbb{D}^{n}$ and $k=1, \ldots, n$, the following inequalities hold:

(a) $\frac{1}{2} \log \frac{1+\left|z_{k}\right|}{1-\left|z_{k}\right|} \leq \omega(z)$.

(b) $\rho(0, z) \leq \frac{1}{2} \sum_{k=1}^{n} \log \frac{1+\left|z_{k}\right|}{1-\left|z_{k}\right|}$.

Proof. To prove (a), fix $z \in \mathbb{D}^{n}$ and $k=1, \ldots, n$, and for $w \in \mathbb{D}^{n}$, define

$$
h(w)=\frac{1}{2} \log \frac{\left|z_{k}\right|+w_{k} \overline{z_{k}}}{\left|z_{k}\right|-w_{k} \overline{z_{k}}}
$$

where Log denotes the principal branch of the logarithm. Then $h \in H\left(\mathbb{D}^{n}\right), h(0)=$ $0, \frac{\partial h}{\partial z_{j}}(w)=0$ for $j \neq k$, and $\frac{\partial h}{\partial z_{k}}(w)=\frac{\overline{z_{k}}\left|z_{k}\right|}{\left|z_{k}\right|^{2}-w_{k}^{2} \bar{z}_{k}^{2}}$. By Theorem 3.3 of [7], $\beta_{h}(w)=$ $\frac{\left(1-\left|w_{k}\right|^{2}\right)\left|z_{k}\right|^{2}}{\left.|| z_{k}\right|^{2}-w_{k}^{2} \bar{z}_{k}^{2}} \leq 1$. In particular,

$$
\frac{1}{2} \log \frac{1+\left|z_{k}\right|}{1-\left|z_{k}\right|}=|h(z)| \leq \omega(z)
$$


To prove (b), observe that for $z \in \mathbb{D}^{n}$ and $u \in \mathbb{C}^{n}$,

$$
H_{z}(u, \bar{u})=\sum_{k=1}^{n} \frac{\left|u_{k}\right|^{2}}{\left(1-\left|z_{k}\right|^{2}\right)^{2}}
$$

(e.g. see [18]), and recall that if $\gamma=\gamma(t)(0 \leq t \leq 1)$ is the geodesic from $w$ to $z$, then

$$
\rho(w, z)=\int_{0}^{1} H_{\gamma(t)}\left(\gamma^{\prime}(t), \overline{\gamma^{\prime}(t)}\right)^{1 / 2} d t .
$$

Since the geodesic from 0 to $z \in \mathbb{D}^{n}$ is parametrized by $\gamma(t)=t z$, for $0 \leq t \leq 1$, we obtain

$$
\begin{aligned}
\rho(0, z) & =\int_{0}^{1}\left(\sum_{k=1}^{n} \frac{\left|z_{k}\right|^{2}}{\left(1-\left|z_{k}\right|^{2} t^{2}\right)^{2}}\right)^{1 / 2} d t \leq \int_{0}^{1} \sum_{k=1}^{n} \frac{\left|z_{k}\right|}{1-\left|z_{k}\right|^{2} t^{2}} d t \\
& =\frac{1}{2} \sum_{k=1}^{n} \log \frac{1+\left|z_{k}\right|}{1-\left|z_{k}\right|} . \quad \square
\end{aligned}
$$

Theorem 3.5. Let $\psi \in H\left(\mathbb{D}^{n}\right)$. Then the following are equivalent:

(a) $M_{\psi}$ is bounded on $\mathcal{B}\left(\mathbb{D}^{n}\right)$.

(b) $M_{\psi}$ is bounded on $\mathcal{B}_{0^{*}}\left(\mathbb{D}^{n}\right)$.

(c) $\psi \in H^{\infty}\left(\mathbb{D}^{n}\right)$ and $\sup _{z \in \mathbb{D}^{n}} \sum_{k=1}^{n} \log \frac{1+\left|z_{k}\right|}{1-\left|z_{k}\right|} Q_{\psi}(z)$ is finite.

Proof. The equivalence $(a) \Longleftrightarrow(c)$ follows from Theorem 3.2 and Lemma 3.4(a). The implication $(c) \Longrightarrow(b)$ follows from Lemma 3.4(b) and (2). To prove that $(b) \Longrightarrow(c)$, suppose $M_{\psi}$ is bounded on $\mathcal{B}_{0^{*}}(D)$. By Theorem 3.2, we have $\psi \in$ $H^{\infty}\left(\mathbb{D}^{n}\right) \cap \mathcal{B}_{0^{*}}\left(\mathbb{D}^{n}\right)$. Thus, fixing $k=1, \ldots, n$, it suffices to show that for a fixed $k=1, \ldots, n, \sup _{z \in \mathbb{D}^{n}} \log \frac{1+\left|z_{k}\right|}{1-\left|z_{k}\right|} Q_{\psi}(z)$ is finite. Fix $w \in \mathbb{D}^{n}$ and for $z \in \mathbb{D}^{n}$ define

$$
f_{w}(z)=\frac{1}{2} \log \frac{1+\overline{w_{k}} z_{k}}{1-\overline{w_{k}} z_{k}} .
$$

It is clear that that $f_{w}(0)=0, \frac{\partial f_{w}}{\partial z_{j}}(z)=0$ for all $j \neq k$, and $\frac{\partial f_{w}}{\partial z_{k}}(z)=\frac{\overline{w_{k}}}{1-\overline{w_{k}} z_{k}^{2}}$. Using Theorem 3.3 of [7, we obtain

$$
Q_{f_{w}}(z)=\frac{\left(1-\left|z_{k}\right|^{2}\right)\left|w_{k}\right|}{\left|1-\bar{w}^{2} z_{k}^{2}\right|} \leq\left|w_{k}\right| \frac{1-\left|z_{k}\right|^{2}}{1-\left|w_{k}\right|^{2}\left|z_{k}\right|^{2}} .
$$

By elementary calculus, one can verify that the function $g(x)=\frac{1-x^{2}}{1-\alpha x^{2}}$ attains its maximum on the interval $[0,1]$ at $x=0$, for $0<\alpha<1$. Thus ||$f_{w} \|_{\mathcal{B}} \leq\left|w_{k}\right|<1$. Furthermore, $f_{w} \in \mathcal{B}_{0^{*}}\left(\mathbb{D}^{n}\right)$ since

$$
\lim _{z \rightarrow \partial^{*} D} Q_{f_{w}}(z) \leq\left|w_{k}\right| \lim _{\left|z_{k}\right| \rightarrow 1^{-}} \frac{1-\left|z_{k}\right|^{2}}{1-\left|w_{k}\right|^{2}\left|z_{k}\right|^{2}}=0 .
$$

Therefore

$$
\left|f_{w}(z)\right| Q_{\psi}(z) \leq \omega_{0}(z) Q_{\psi}(z) \leq \sigma_{0, \psi}
$$


which is finite by Theorem $3.2(\mathrm{~b})$. Observe that

$$
\begin{aligned}
\left|f_{w}(z)\right| & =\frac{1}{2}\left|\log \frac{1+\overline{w_{k}} z_{k}}{1-\overline{w_{k}} z_{k}}\right| \geq \frac{1}{2}\left(\log \left|\frac{1+\overline{w_{k}} z_{k}}{1-\overline{w_{k}} z_{k}}\right|-\operatorname{Arg}\left(\frac{1+\overline{w_{k}} z_{k}}{1-\overline{w_{k}} z_{k}}\right)\right) \\
& \geq \frac{1}{2}\left(\log \left|\frac{1+\overline{w_{k}} z_{k}}{1-\overline{w_{k}} z_{k}}\right|-\frac{\pi}{2}\right),
\end{aligned}
$$

where Arg denotes the principal value of the argument. Thus

$$
\frac{1}{2} \log \left|\frac{1+\overline{w_{k}} z_{k}}{1-\overline{w_{k}} z_{k}}\right| \leq\left|f_{w}(z)\right|+\frac{\pi}{4} .
$$

For $w_{k}=r_{k} e^{i \theta_{k}}$ where $\theta_{k}=\arg \left(z_{k}\right)$, using (6), we obtain

$$
\frac{1}{2} \log \frac{1+r_{k}\left|z_{k}\right|}{1-r_{k}\left|z_{k}\right|} Q_{\psi}(z) \leq \sigma_{0, \psi}+\frac{\pi}{4} \beta_{\psi} .
$$

Letting $r_{k} \rightarrow 1$, we obtain $\sup _{z \in \mathbb{D}^{n}} \log \frac{1+\left|z_{k}\right|}{1-\left|z_{k}\right|} Q_{\psi}(z)<\infty$, completing the proof.

Finally, we provide sufficient conditions for the boundedness of $M_{\psi}$ on $\mathcal{B}(D)$ to be equivalent to the boundedness on $\mathcal{B}_{0^{*}}(D)$, where $D$ is any bounded homogeneous domain in $\mathbb{C}^{n}$. The notation $A \asymp B$ means there exist constants $c_{1}, c_{2}>0$ such that $c_{1} A \leq B \leq c_{2} A$.

Proposition 3.6. Let $D$ be a bounded homogeneous domain in $\mathbb{C}^{n}$ and $\psi \in H(D)$. If $\sigma_{\psi} \asymp \sigma_{0, \psi}$ and $\lim _{z \rightarrow \partial^{*} D} \omega_{0}(z)=\infty$, then $M_{\psi}$ is bounded on $\mathcal{B}(D)$ if and only if it is bounded on $\mathcal{B}_{0^{*}}(D)$.

Proof. First, assume $M_{\psi}$ is bounded on $\mathcal{B}(D)$. Then, $\psi \in H^{\infty}(D)$ and $\sigma_{0, \psi}$ is finite. From the hypothesis, it follows that $Q_{\psi}(z) \rightarrow 0$ as $z \rightarrow \partial^{*} D$. Thus, $\psi \in \mathcal{B}_{0^{*}}(D)$, proving that $M_{\psi}$ is bounded on $\mathcal{B}_{0^{*}}(D)$. Conversely, if $M_{\psi}$ is bounded on $\mathcal{B}_{0^{*}}(D)$, it follows immediately that $M_{\psi}$ is bounded on $\mathcal{B}(D)$, since $\sigma_{\psi} \asymp \sigma_{0, \psi}$.

\section{Operator Norm Estimates}

In this section, we provide estimates on the norm of the bounded multiplication operators on the Bloch space and *-little Bloch space of a bounded homogeneous domain. These estimates correspond to those established in [1] for the case of the unit disk.

Theorem 4.1. Let $D$ be a bounded homogeneous domain in $\mathbb{C}^{n}$.

(a) If $\psi \in H(D)$ induces a bounded multiplication operator on $\mathcal{B}(D)$, then

$$
\max \left\{\|\psi\|_{\mathcal{B}},\|\psi\|_{\infty}\right\} \leq\left\|M_{\psi}\right\| \leq \max \left\{\|\psi\|_{\mathcal{B}},\|\psi\|_{\infty}+\sigma_{\psi}\right\} .
$$

(b) If $\psi \in H(D)$ induces a bounded multiplication operator on $\mathcal{B}_{0^{*}}(D)$, then

$$
\max \left\{\|\psi\|_{\mathcal{B}},\|\psi\|_{\infty}\right\} \leq\left\|M_{\psi}\right\| \leq \max \left\{\|\psi\|_{\mathcal{B}},\|\psi\|_{\infty}+\sigma_{0, \psi}\right\} .
$$

Proof. We will prove the norm estimates for $M_{\psi}$ bounded on $\mathcal{B}(D)$, the argument for $\mathcal{B}_{0^{*}}(D)$ is analogous. By Lemma 11 of [1], we have $\|\psi\|_{\infty} \leq\left\|M_{\psi}\right\|$. Furthermore, for $f$ identically $1,\left\|M_{\psi} f\right\|_{\mathcal{B}}=\|\psi\|_{\mathcal{B}}$. Therefore $\left\|M_{\psi}\right\| \geq \max \left\{\|\psi\|_{\mathcal{B}},\|\psi\|_{\infty}\right\}$.

Next, let $f \in \mathcal{B}(D)$. and apply the identity $|f(0)|=\|f\|_{\mathcal{B}}-\beta_{f}$ to (4) to deduce

$$
\left\|M_{\psi} f\right\|_{\mathcal{B}} \leq\|\psi\|_{\mathcal{B}}\|f\|_{\mathcal{B}}+\left(\|\psi\|_{\infty}+\sigma_{\psi}-\|\psi\|_{\mathcal{B}}\right) \beta_{f}
$$

If $\|\psi\|_{\infty}+\sigma_{\psi} \leq\|\psi\|_{\mathcal{B}}$, then $\left\|M_{\psi} f\right\|_{\mathcal{B}} \leq\|\psi\|_{\mathcal{B}}\|f\|_{\mathcal{B}}$, while if $\|\psi\|_{\infty}+\sigma_{\psi} \geq\|\psi\|_{\mathcal{B}}$, then $\left\|M_{\psi} f\right\|_{\mathcal{B}} \leq\left(\|\psi\|_{\infty}+\sigma_{\psi}\right)\|f\|_{\mathcal{B}}$, proving the upper estimate. 


\section{Spectrum}

In this section, we determine the spectra of the bounded multiplication operators on the Bloch space and $*$-little Bloch space of a bounded homogeneous domain in $\mathbb{C}^{n}$, thereby extending the results obtained in [1] for the case of the unit disk.

Recall that the spectrum of an operator $T$ is defined as

$$
\sigma(T)=\{\lambda \in \mathbb{C}: T-\lambda I \text { is not invertible }\},
$$

where $I$ is the identity operator. If $\lambda \in \mathbb{C}$, then $M_{\psi}-\lambda I=M_{\psi-\lambda}$. Thus, $\lambda \in \sigma\left(M_{\psi}\right)$ if and only if $M_{\psi-\lambda}$ is not invertible.

Theorem 5.1. Let $D$ be a bounded homogeneous domain in $\mathbb{C}^{n}$ and assume $\psi \in$ $H(D)$ induces a bounded multiplication operator on $\mathcal{B}(D)$ or $\mathcal{B}_{0^{*}}(D)$. Then $\sigma\left(M_{\psi}\right)=$ $\overline{\psi(D)}$.

Proof. We first prove the result for $M_{\psi}$ acting on $\mathcal{B}(D)$. Let $\lambda \in \psi(D)$. Then there exists $z_{0} \in D$ at which the function $(\psi(z)-\lambda)^{-1}$ is singular. Thus $M_{\psi-\lambda}$ is not invertible and $\psi(D) \subseteq \sigma\left(M_{\psi}\right)$. Since the spectrum is closed, $\overline{\psi(D)} \subseteq \sigma\left(M_{\psi}\right)$.

Suppose $\lambda \notin \overline{\psi(D)}$. Then $\psi(z)-\lambda$ is bounded away from zero; that is, there exists $\alpha>0$ such that $|\psi(z)-\lambda| \geq \alpha$ for all $z \in D$. Thus the function $g$ defined by $g(z)=(\psi(z)-\lambda)^{-1}$ is bounded holomorphic on $D$. By Theorem 3.2 , the multiplication operator induced by $g$ is bounded on $\mathcal{B}(D)$ since

$$
\sigma_{g}=\sup _{z \in D} \omega(z) Q_{g}(z) \leq \sup _{z \in D} \frac{1}{\alpha^{2}} \omega(z) Q_{\psi}(z)=\frac{1}{\alpha^{2}} \sigma_{\psi}<\infty .
$$

Thus $\lambda \notin \sigma\left(M_{\psi}\right)$.

To prove the result for $M_{\psi}$ acting on $\mathcal{B}_{0^{*}}(D)$, it suffices to show that the bounded holomorphic function $g$ defined above is in the $*$-little Bloch space for $\lambda \notin \overline{\psi(D)}$. Since $\psi \in \mathcal{B}_{0^{*}}(D)$ and $Q_{g}(z) \leq \frac{1}{\alpha^{2}} Q_{\psi}(z)$, it follows that $Q_{g}(z) \rightarrow 0$ as $z \rightarrow \partial^{*} D$, as desired.

\section{Compactness}

In this section, we characterize the compact multiplication operators on the Bloch space and the $*$-little Bloch space of a bounded homogeneous domain in $\mathbb{C}^{n}$. The key to this characterization is the spectral theorem of compact operators due to Riesz (see Theorem 7.1 of [8]). If we apply Theorem 5.1 to the spectral theorem of compact operators, we obtain the following lemma.

Lemma 6.1. Let $D$ be a bounded homogeneous domain in $\mathbb{C}^{n}$ and $\psi \in H(D)$ such that $M_{\psi}$ is bounded on $\mathcal{B}(D)$ or $\mathcal{B}_{0^{*}}(D)$. Then $\overline{\psi(D)}$ is non-empty and at most countably infinite. Furthermore, if $\overline{\psi(D)}$ is a singleton, then $\overline{\psi(D)}=\{0\}$.

Theorem 6.2. Let $D$ be a bounded homogeneous domain. The only compact multiplication operator on $\mathcal{B}(D)$ or $\mathcal{B}_{0^{*}}(D)$ is the operator whose symbol is identically 0.

Proof. Let $D$ be a bounded homogeneous domain and $\psi \in H(D)$. Clearly if $\psi \equiv 0$, then $M_{\psi}$ is compact on $\mathcal{B}(D)$ or $\mathcal{B}_{0^{*}}(D)$. Suppose $M_{\psi}$ is compact on $\mathcal{B}(D)$ or $\mathcal{B}_{0^{*}}(D)$. By Lemma 6.1, the range of $\psi$ is at most countable. On the other hand, if the range of $\psi$ contains two distinct points, then it contains a continuum, and thus is uncountable. Hence, the range of $\psi$ must be a single point and by Lemma 6.1, $\psi$ must be identically 0 . 


\section{ISOMETRIES}

In this section, we characterize the isometric multiplication operators on the Bloch space of a large class of bounded symmetric domains in $\mathbb{C}^{n}$.

Remark 2. First, we observe that if $M_{\psi}$ is an isometry on $\mathcal{B}(D)$ for any bounded homogeneous domain $D$, then $\left\|M_{\psi}\right\|=\|\psi\|_{\mathcal{B}}$. This follows immediately by applying $M_{\psi}$ to the constant function 1 . In fact, if $\psi$ is any constant function of modulus one, then $M_{\psi}$ is an isometry on the Bloch space. Of course, the same is true on the $*$-little Bloch space.

In 6], Cohen and the second author defined the Bloch constant of a bounded homogeneous domain $D$ in $\mathbb{C}^{n}$ as

$$
c_{D}=\sup \left\{\beta_{f}: f \in H(D), f(D) \subseteq \mathbb{D}\right\} .
$$

When $D$ is a Cartan classical domain, the Bloch constants were computed to be

$$
c_{D}= \begin{cases}\sqrt{2 /(n+m)}, & \text { if } D \in R_{I}, \\ \sqrt{2 /(n+1)}, & \text { if } D \in R_{I I}, \\ \sqrt{1 /(n-1)}, & \text { if } D \in R_{I I I}, \\ \sqrt{2 / n}, & \text { if } D \in R_{I V} .\end{cases}
$$

In [20, Zhang computed the Bloch constant of the two exceptional domains to be $1 / \sqrt{6}$ and $1 / 3$. By Theorem 3 of $[\underline{6}$, extended to include the exceptional domains, if $D=D_{1} \times \cdots \times D_{k}$ is a bounded symmetric domain in standard form, then

$$
c_{D}=\max _{1 \leq j \leq k} c_{D_{j}}
$$

Lemma 7.1. Let $D=D_{1} \times \cdots \times D_{k}$ be a bounded symmetric domain in standard form. Then $c_{D} \leq 1$, and $c_{D}=1$ if and only if $D_{j}=\mathbb{D}$ for some $j \in\{1, \ldots, k\}$.

Proof. First we consider the Bloch constant for each irreducible factor $D_{j}$ for $1 \leq$ $j \leq k$. Using the dimensional restrictions in Section 2 by inspection it is clear that $c_{D_{j}} \leq 1$ and $c_{D_{j}}=1$ if and only if $D_{j}$ is in $R_{I}$ with $n=m=1$. Thus, by (7), $c_{D} \leq 1$ and $c_{D}=1$ if and only if there exists $j \in\{1, \ldots, k\}$ such that $D_{j}$ is in $R_{I}$ with $n=m=1$. If there exists such a $j$, then $D_{j}=\mathbb{D}$.

We denote by $\mathfrak{D}$ the set of bounded symmetric domains $D$ for which $c_{D}<1$. By Lemma 7.1, these are precisely the bounded symmetric domains which do not have $\mathbb{D}$ as a factor when written in standard form. The next result follows immediately from Remark 2 using induction on $k$.

Lemma 7.2. Let $D$ be a bounded homogeneous domain in $\mathbb{C}^{n}$ and $\psi \in H(D)$. If $M_{\psi}$ is an isometry on $\mathcal{B}(D)$ (or $\left.\mathcal{B}_{0^{*}}(D)\right)$, then $M_{\psi^{k}}$ is an isometry on $\mathcal{B}(D)$ (respectively, $\left.\mathcal{B}_{0^{*}}(D)\right)$ for all $k \in \mathbb{N}$. In particular, $\left\|\psi^{k}\right\|_{\mathcal{B}}=1$ for all $k \in \mathbb{N}$.

Lemma 7.3. Let $D$ be a bounded symmetric domain and $\psi \in H(D)$. If $M_{\psi}$ is an isometry on $\mathcal{B}(D)$ or $\mathcal{B}_{0^{*}}(D)$, then $\beta_{\psi^{k}} \leq c_{D}$ for all $k \in \mathbb{N}$. In particular, if $D \in \mathfrak{D}$, then $\beta_{\psi^{k}}<1$ for all $k \in \mathbb{N}$.

Proof. By Lemma 11 of [1], $\left\|\psi^{k}\right\|_{\infty} \leq\left\|M_{\psi^{k}}\right\|=1$. Thus $\psi^{k}$ is either a constant function of modulus one or a bounded holomorphic function mapping into $\mathbb{D}$. If $\psi^{k}$ is a constant function of modulus one, then $\beta_{\psi^{k}}=0$. If $\psi^{k}$ is bounded holomorphic mapping into $\mathbb{D}$, then the definition of the Bloch constant of $D$ gives $\beta_{\psi^{k}} \leq c_{D}$ for 
all $k \geq 1$. Thus in both cases, $\beta_{\psi^{k}} \leq c_{D}$ for all $k \in \mathbb{N}$. If $D \in \mathfrak{D}$, the conclusion follows from Lemma 7.1 .

Theorem 7.4. Let $D \in \mathfrak{D}$ and $\psi \in H(D)$. Then $M_{\psi}$ is an isometry on $\mathcal{B}(D)$ or $\mathcal{B}_{0^{*}}(D)$ if and only if $\psi$ is a constant function of modulus one.

Proof. We will prove this statement for $\mathcal{B}(D)$ (the proof follows the same argument for $\left.\mathcal{B}_{0^{*}}(D)\right)$. Since the multiplication operators induced by unimodular constants are isometries, we only need to prove the converse. Suppose $M_{\psi}$ is an isometry on $\mathcal{B}(D)$ and $\psi$ is not a constant function of modulus one. Then $\psi(0)=a$, for some $|a|<1$. Since $\left\|\psi^{k}\right\|_{\mathcal{B}}=1$, by Lemma 7.3 we have

$$
|a|^{k}=1-\beta_{\psi^{k}} \geq 1-c_{D}
$$

so that $|a|^{k}$ is bounded away from 0 , contradicting the fact that $|a|^{k} \rightarrow 0$ as $k \rightarrow \infty$. Thus, if $M_{\psi}$ is an isometry, then $\psi$ must be a constant function of modulus one.

\section{Open Questions}

Let $D$ be a bounded homogeneous domain.

(1) If $\psi \in H(D)$ induces a bounded multiplication operator on $\mathcal{B}(D)$, are the upper bounds of Theorem 4.1 sharp?

(2) If $D$ is not conformally equivalent to the unit ball and $\rho$ is the Bergman distance on $D$, is $\omega(z)=\rho(0, z)$ for each $z \in D$ ?

(3) If $D$ is a bounded homogeneous domain other than the ball:

(a) Is $\omega(z)=\omega_{0}(z)$ for each $z \in D$ ?

(b) Is $\sigma_{\psi} \asymp \sigma_{0, \psi}$ for some $\psi \in H(D)$ ? If so, which functions $\psi$ satisfy this property?

(c) Is $\lim _{z \rightarrow \partial^{*} D} \omega_{0}(z)=\infty$ ?

Remark 3. Theorem 7.4 pertains to bounded symmetric domains which do not have $\mathrm{D}$ as a factor, and so it does not apply to the case of the Bloch space on the unit disk. However, in [1, the authors showed that even in this case the isometric multiplication operators on the Bloch space of $\mathbb{D}$ are precisely those operators whose symbol is a constant function of modulus one. This result was known to N. Zorboska, R. Zhao and Z. Cuckovic. Thus we end this paper with the following conjecture.

Conjecture. Let $D$ be a bounded homogeneous domain. Then $M_{\psi}$ is an isometry on $\mathcal{B}(D)$ or $\mathcal{B}_{0^{*}}(D)$ if and only if $\psi$ is a constant function of modulus one.

\section{REFERENCES}

1. R. F. Allen and F. Colonna, Isometries and spectra of multiplication operators on the Bloch space, Bull. Austral. Math. Soc., to appear.

2. - On the isometric composition operators on the Bloch space in $\mathbb{C}^{n}$, J. Math. Anal. Appl., to appear.

3. J. Arazy, Multipliers of Bloch functions, University of Haifa Mathematics Publications 54 (1982).

4. L. Brown and A. L. Shields, Multipliers and cyclic vectors in the Bloch space, Michigan Math. J. 38 (1991), 141-146.

5. E. Cartan, Sur les domains bournés de l'espace de $n$ variable complexes (French), Abh. Math. Sem. Univ. Hamburgh 11 (1935), 116-162.

6. J. M. Cohen and F. Colonna, Bounded holomorphic functions on bounded symmetric domains, Trans. Amer. Math. Soc. 343 (1994), 135-156. 
7. - Isometric composition operators on the Bloch space in the polydisk, Contemp. Math. 454 (2008), 9-21.

8. J. B. Conway, A Course in Functional Analysis, Second Edition, Springer-Verlag, New York, 1990.

9. C. Cowen and B. MacCluer, Composition Operators on Spaces of Analytic Functions, CRC Press, Boca Raton, 1995.

10. D. Drucker, Exception Lie algebras and the structure of Hermitian symmetric spaces, Mem. Amer. Math. Soc. 208 (1978), 1-207.

11. P. L. Duren, B. W. Romberg and A. L. Shields, Linear functions on $H^{p}$ spaces with $0<p<1$, J. Reine Angew. Math. 238 (1969), 32-60.

12. K. T. Hahn, Holomorphic mappings of the hyperbolic space into the complex Euclidean space and the Bloch theorem, Canad. J. Math. 27 (1975), 446-458.

13. S. Kobayashi, Hyperbolic Manifolds and Holomorphic Mappings: An Introduction, Second Edition, World Scientific, London, 2005.

14. S. Krantz and D. Ma, Bloch functions on strongly pseudoconvex domains, Indiana Univ. Math. J. 37 (1988), 145-163.

15. S. Ohno and R. Zhao, Weighted composition operators on the Bloch space, Bull. Austral. Math. Soc 63 (2001), 177-185.

16. I. I. Pjateckiı̌-S̆apiro, On a problem proposed by E. Cartan (Russian), Dokl. Akad. Nauk SSSR 124 (1959), 272-273

17. J. H. Shapiro, Composition Operators and Classical Function Theory, Springer-Verlag, New York, 1993.

18. R. M. Timoney, Bloch functions in several complex variables I, Bull. London Math. Soc. 12 (1980), 241-267.

19. $\longrightarrow$ Bloch functions in several complex variables II, J. Reine Angew. Math. 319 (1980), $1-22$.

20. G. Zhang, Bloch constants of bounded symmetric domains, Trans. Amer. Math. Soc. 349 (1997), 2941-2949.

21. Z. Zhou and R. Chen, Weighted composition operators from $F(p, q, s)$ to Bloch type spaces on the unit ball (preprint) (http://arxiv.org/abs/math/0503614v9).

22. Z. Zhou and R. Chen, Weighted composition operators between different Bloch-type Spaces in polydisk (preprint) (http://arxiv.org/abs/math/0503622v2).

23. K. Zhu, Spaces of Holomorphic Functions in the Unit Ball, Springer-Verlag, New York, 2004.

Department of Mathematical Sciences, George Mason University, Fairfax, VA 22030

E-mail address: rallen2@gmu.edu

Department of Mathematical Sciences, George Mason University, Fairfax, VA 22030

E-mail address: fcolonna@gmu.edu 